\title{
Design and real time implementation of single phase boost power factor correction converter
}

\author{
Amar Bouafassa ${ }^{\text {a,*, }}$, Lazhar Rahmani ${ }^{\mathrm{a}}$, Saad Mekhilef ${ }^{\mathrm{b}}$ \\ a Laboratoire d'automatique de Sétif (LAS), Département d'électrotechnique, Faculté de Technologie, Université Sétif-1, Algérie
}

Department of Electrical Engineering, Faculty of Engineering, University of Malaya, 50603 Kuala Lumpur, Malaysia

A R T I C L E I N F O

Article history:

Received 5 May 2014

Received in revised form

24 September 2014

Accepted 10 October 2014

Available online 31 October 2014

Keywords:

Power factor correction

Higher order sliding mode controlle

Predictive control

AC-DC Boost converter

dSPACE 1104

\section{Introduction}

Recently, the power electronics components have gained popularity in the development of new static converters to supply domestic and industrial applications due to the introduction of the fast switches components and appearance of new types of control techniques. Moreover, the use of power converters for renewable energy (solar, wind) becomes more important in the world due to the energy crisis. The AC-DC converter is an important element of the power supplies. Indeed, it has numerous applications in different power levels; low power like chargers of mobile phones that need a few watts, high power like electric welding that need a few $\mathrm{kW}$. The AC-DC converter includes various topologies such as; buck, boost and buck-boost, etc. $[1,2]$. Among these types, the AC-DC boost converter has interesting features, which are the lifting operation, simple structure and low cost. The development of semiconductor allows the appearance of new power converters use a switch mode operation. The nonlinear loads and switching losses lead to more energy losses. Furthermore, Due to increasingly use of boost converter in the large electronic equipment, intensive efforts have been devoted to adopt international standard such as IEC 1000-3-2, EN61000-3-2 in Europe and the IEEE 519 in USA [3]. To improve the power factor in the boost converter topology, there are two types of power factor correction methods, passive method used for old

\footnotetext{
${ }^{*}$ Corresponding author

E-mail addresses: amar.bouafassa@gmail.com (A. Bouafassa), lazhar_rah@yahoo.fr (L. Rahmani), saad@um.edu.my (S. Mekhilef).

http://dx.doi.org/10.1016/j.isatra.2014.10.004

0019-0578/0 2014 ISA. Published by Elsevier Ltd. All rights reserved.
}

power converters, and active method used recently in the most of electronics components that shown in Fig. 1. Several researches have been done to improve and find an adequate control for the PFC converter, which can provide a better performance.

Commonly, the traditional regulators like PI and PID have been used to regulate the output voltage of the boost converter [4,5]. These types of controls are based on modeling of the system around a nominal point under constant parameters and disturbance, which provide an acceptable performance but if the parameters change, the system loses its performance and give a bad results. For this reason, various intelligent controllers have been introduced to get an optimal performance of the converter regardless of parameters variations. Among these controllers fuzzy logic [6,7], sliding mode [8-10], predictive technique [11,12], artificial neural network [13] are used.

The sliding mode control has attracted great research interest [14-17]. Several researches have used SMC for the control of nonlinear and multivariable systems due to its disturbance rejection [18-20]. However, the drawback of sliding mode controller is the high frequency oscillations (chattering phenomenon), which is a major obstacle for the implementation of standard SMC. For these reasons. The HOSMC is proposed to overcomes the main drawbacks of classical SMC, its provides a smooth control, good performance yielding to less chattering in real time implementation, and better convergence while preserving the robustness properties [21-24]. Among the existing algorithms, the super twisting algorithms has been designed and evaluated in this paper. It has interesting features and maintains the distinctive performances of the classical SMC. It provides a systematic method to 

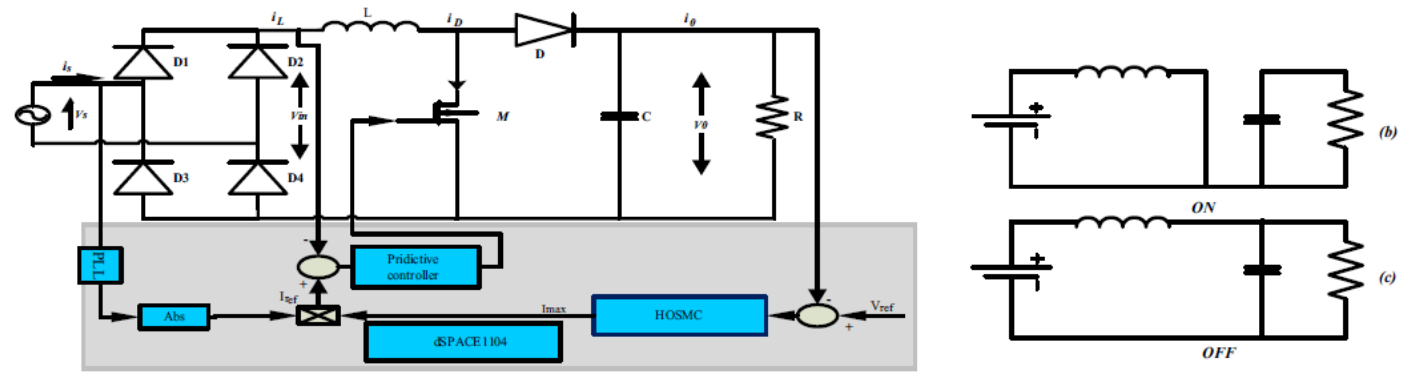

Fig. 1. PFC pre-regulator

solve the stability and modeling imprecision problems in new electronic components. Moreover, his practical implementation is simple and assure in both the accuracy and convergence in finite time. Similarly, the predictive control has been employed in the industrial applications those need high specific performance, due to its reliability and disturbance attenuation $[25,26]$. The predictive technique has been used in this work to investigate its speed and implementation simplicity, which leads a fast dynamic response and better efficiency.

Generally, fast settling time leads to large overshoot; it is well known that these two important requirements are the most common problems in controller design, which are not desired in many practical applications. Hence the user is compelled to choose either fast response or low overshoot. This particular problem can be solved by using the proposed method. The main contribution of this paper is the real time implementation on dSPACE 1104 of a new robust hybrid controller, composed by HOSMC based on super twisting in the DC voltage loop, and predictive controller in the current loop, which takes into account a better steady state performance and transient response. To verify the controllers performance for steady and transient states, at first simulations have been carried out under different loading conditions along with different reference output voltages. Later an experimental test using the dSPACE 1104 board has been conducted to verify the simulations results. From both simulation and experimental results, the robustness of the proposed controller based boost converter for PFC has been verified and evaluated.

The paper is organized as follows. Section 2 describes the proposed control methods for the boost converter. Sections 3 and 4 present the simulation and the experimental results along with the discussion. In Section 5 a general conclusion has been drawn by focusing the significant point of the paper.

\section{Description of proposed control method}

\subsection{Modeling of the system}

The basic model of the boost converter is defined according to the state of switch $M$. When the switch $M$ is turned on $(u=1)$, the voltage across the transistor is equal to zero and the diode is closed. Fig. 1b shows the equivalent circuit of the boost converter in the ON state. As soon as the switch M is turned off $(u=0)$, the voltage across the diode is equal to zero, Fig. 1c shows the equivalent circuit in this operation mode [4]. The state space model for the boost converter governed the real switched system can be expressed as follow [27].

$\left\{\begin{array}{c}C \frac{d V_{0}}{d t}=(1-u) i_{L}-i_{0} \\ L \frac{d i_{1}}{d t}=V_{i n}-(1-u) V_{0}\end{array}\right.$
When a non-controlled rectifier is connected to the source voltage to ensure the converted AC/DC as shown in Fig. 1, the current drawn from the source $i_{s}$ will be very distorted (high THD) and not in phase with the input voltage $V s$, which increases the reactive power and lead to a low input power factor. To solve this problem, one possibility is to add a PFC circuit using two control loops. The dc-bus voltage is sensed and compared with its reference value $V_{\text {ref. }}$. The obtained error is used as input for the voltage loop controller, the output of the voltage loop controller $I_{\max }$ multiplied by Isin $\omega$ tl obtained from PLL is the reference current $i_{\text {ref, }}$, the reference current is compared with the inductor current $i_{L}$. The obtained error is used as input for the current loop controller that calculate the duty cycle.

\subsection{Voltage loop controller}

To regulate the output voltage, the high order sliding mode controller (the second) based on super twisting algorithm has been used which has a specific operating mode. A sliding mode is said " $r$ th order sliding mode" if $S(\mathrm{t})=\mathrm{S}(\mathrm{t})=\ldots=\mathrm{S}^{(\mathrm{r}-1)}=0$. In HOSMC, the idea is to force the error to move on the switching surfaces and to keep its $(r-1)$ first successive derivatives null. More specifically, SOSMC aims for $\mathrm{S}(\mathrm{t})=\dot{\mathrm{S}}(\mathrm{t})=0$, which is, the controller's aim to steer to zero at the intersection of $\mathrm{S}(\mathrm{t})$ and $\mathrm{S}(t)$ in the state space [28]. The main feature of the proposed method is that the system present a high robustness performance during the change of the parameters. The control is carried out based on the state variables used to build a switching surface, whose purpose is to force the dynamic system to follow this switching surface in finite time and reduce chattering effects.

Let us the following tracking error as:

$$
e(t)=V_{r e f}-V_{0}
$$

The aim of control is that:

$\lim _{t \rightarrow \infty}\|e(t)\|=\lim _{t \rightarrow \infty}\left\|V_{r e f}(t)-V_{0}(t)\right\|=0$

Then we will have:

$\frac{d e(t)}{d t}=\frac{d V_{r e f}(t)}{d t}-\frac{d V_{0}(t)}{d t}=\frac{d V_{r e f}(t)}{d t}-\frac{1}{C}\left(i_{L}-\frac{V_{0}}{R}\right)$

Thus we have

$\frac{d^{2} e(t)}{d t^{2}}=\frac{d^{2} V_{r e f}(t)}{d t^{2}}-\frac{d^{2} V_{0}(t)}{d t^{2}}=\frac{d^{2} V_{r e f}(t)}{d t^{2}}-\frac{1}{C}\left(\frac{d i_{L}}{d t}-\frac{1}{R} \frac{d V_{0}}{d t}\right)$

Using Eq. (1) and Eq. (5), the second derivative rewrite as follow:

$\frac{d^{2} e(t)}{d t^{2}}=\frac{d^{2} V_{r e f}(t)}{d t^{2}}-\frac{1}{C}\left(\frac{1}{L}\left(V_{i n}-V_{0}\right)-\frac{1}{R C}\left(i_{L}-\frac{V_{0}}{R}\right)\right)$ 
Let us consider the following surface [23].

$S(e, t)=\left(\frac{\partial}{\partial t}+\lambda\right)^{(n-1)} e(t)$

Where $\lambda$ is a positive constant. It is clear that the degree related to the voltage in Eq. (1) is equal to 1; in this case the surface is chosen as the setting error. Therefore, the surface can be writing as

$S(t)=V_{\text {ref }}-V_{0}$

To maintain the trajectory of the output voltage $\left(V_{0}\right)$ in switching surface, an equivalent current $\left(I_{\text {eq }}\right)$ control is applied considering the following invariance conditions:

$\left\{\begin{array}{l}S(t)=V_{\text {ref }}-V_{0}=0 \\ \dot{S}(t)=\frac{d V_{\text {ref }}(t)}{d t}-\frac{d V_{g}(t)}{d t}=0\end{array}\right.$

To prove the stability and ensure the stable convergence property of the proposed controller, the Lyapunov function is selected as

$V(t)=\frac{1}{2} S^{2}(t)+\frac{1}{2} \dot{S}^{2}(t)$

Where $\mathrm{V}(0)=0, \mathrm{~V}(\mathrm{t})>0$ for $\mathrm{S}(\mathrm{t}) \neq 0$ and $\dot{\mathrm{S}}(t) \neq 0$

The stability is ensured if the derivative of the Lyapunov function is negative, and satisfies the following condition:

$\dot{V}(t)<0, \quad S(t) \neq 0, \quad \dot{S}(t) \neq 0$

Taking the first derivative of Eq. (10) yields

$\dot{V}(t)=S(t) \dot{S}(t)+\dot{S}(t) \ddot{S}(t)=\dot{S}(t) \cdot[S(t)+\ddot{S}(t)]$

Using Eqs.(2) and (6) yields

$$
\begin{aligned}
\dot{V}(t)= & \dot{S}(t)\left[V_{\text {ref }}-V_{0}+\ddot{V}_{\text {ref }}-\frac{1}{C}\left(\frac{1}{L}\left(V_{\text {in }}-V_{0}\right)-\frac{1}{R C}\left(i_{L}-\frac{V_{0}}{R}\right)\right)\right] \\
= & {\left[\dot{V}_{\text {ref }}-\frac{1}{C}\left(i_{L}-\frac{V_{0}}{R}\right)\right] } \\
& .\left[V_{\text {ref }}-V_{0}+\ddot{V}_{\text {ref }}-\frac{1}{C}\left(\frac{1}{L}\left(V_{\text {in }}-V_{0}\right)-\frac{1}{R C}\left(i_{L}-\frac{V_{0}}{R}\right)\right)\right]
\end{aligned}
$$

For our case, $V_{r e f}$ is constant and its derivative is null, Eq.(13) can be represented as

$\dot{V}(t)=-\frac{1}{C}\left(i_{L}-\frac{V_{0}}{R}\right)\left[V_{\text {ref }}-V_{0}-\frac{1}{C}\left(\frac{1}{L}\left(V_{\text {in }}-V_{0}\right)-\frac{1}{R C}\left(i_{L}-\frac{V_{0}}{R}\right)\right)\right]<0$

when the system is in the reaching phase with $\mathrm{S}(\mathrm{t}) \neq 0, \dot{\mathrm{S}}(t)$ and have $V(t)$ is negative definite. From the above analysis, the derivative of the Lyapunov function is a negative definite, the system is globally asymptotically stable.

The global control is composed of the equivalent control $\left(I_{\mathrm{eq}}\right)$ and the super twisting algorithm terms. Now, lets us the super twisting algorithm, this later is used in order to stabilize the system, avoid chattering effects, and converges the system to the desired trajectory in finite time. The advantage of super twisting algorithm is that; it does not need any information on the time derivative of the sliding variable and maintains all the distinctive robust features of the SMC. The control law is composed by two parts defined by the following control law:

$u(t)=u_{1}(t)+u_{2}(t)$

where

$\dot{u}_{1}(t)=\{-u$ if $|u|>1-w \operatorname{sign}(S)$ if $|u| \leq 1$

$u_{2}(t)=\left\{-\lambda\left|S_{0}\right|^{\rho} \operatorname{sign}(S) i f|S|>S_{0}-\lambda|S|^{\rho} \operatorname{sign}(S)\right.$ if $|S| \leq S_{0}$

Where $S_{0}$ is a boundary layer around the sliding surface.
If the control gains satisfy the constraint in Eq.(18), the convergence to the sliding surface in finite time is fulfill. The method to calculate of $\Phi, \Gamma_{\mathrm{m}}$ and $\Gamma_{\mathrm{M}}$ has been presented in [21]. $w>\frac{\Phi}{T_{m}}$

$\lambda^{2} \geq \frac{4 \Phi}{\Gamma_{m}^{2}} \Gamma_{M(m+\infty)}$

$0<\rho \leq 0.5$

By adding the super twisting algorithm terms, we obtain the global control of voltage loop as follow:

$I_{\text {Max }}=I_{e q}+\int_{0}^{t} \dot{u}_{1} d t+u_{2}$

\subsection{Current loop controller}

Due to predictive control's suitable performance and flexible implementation on real time, it has been used to control the power converters for last few years [1]. In this work, the predictive method has been used to control converter switching. Taking into account the state of the switch from the circuit shown in Fig. 1, the equations of the inductor current $i_{L}(\mathrm{t})$ for each state can be expressed as [12,29]when $M$ is turned on

$L \frac{d i_{L}}{d t}=V_{i n}(t)$ for $t(k) \leq t<t(k)+d(k) T_{s}$

when $\mathrm{M}$ is turned off

$L \frac{d i_{L}}{d t}=V_{i n}(t)-V_{0}(t)$ for $t(k)+d(k) . T_{s} \leq t<t(k+1)$

Where $V_{\text {in }}(t)$ and $V_{0}(t)$ are the input and output voltage respectively, $t(k)$ and $t(k+1)$ are the started time of the $k$ th and $(k+1)$ th switching cycle respectively, $d(k)$ and $T_{s}$ are the duty cycle and switching period respectively. Since the switching frequency is more than the line frequency, Eq. (20) and Eq. (21) can be rewritten as

$\frac{i_{L}\left(t(k)+d(k) T_{s}\right)-i_{L}(t(k))}{d(k) T_{s}}=V_{\text {in }}(t(k))$

$L \frac{i_{L}(t(k+1))-i_{L}\left(t(k)+d(k) \cdot T_{s}\right)}{(1-d(k)) \cdot T_{s}}=V_{\text {in }}(t(k))-V_{0}(t(k))$

The diagram presented in Fig. 2, represents the inductor current during one switching cycle [12].

At instant $t(k)+d(k) . T_{s}$, the inductor current can be derived from Eq. (22) as

$i_{L}\left(t(k)+d(k) T_{s}\right)=i_{L}(t(k))+\frac{1}{L} V_{i n}(t(k)) d(k) T_{s}$

In the start time of switching cycle $t(k+1)$, the inductor current can be derived from Eq. (23) as follow:

$i_{L}(t(k+1))=i_{L}\left(t(k)+d(k) T_{s}\right)+\frac{1}{L}\left(V_{i n}(t(k))-V_{0}(t(k))\right)(1-d(k)) T_{s}$

Substituting Eqs. (24) and (25), the inductor current can be written as

$i_{L}(t(k+1))=i_{L}(t(k))+\frac{1}{L}\left(V_{i n}(t(k)) T_{s}-\frac{1}{L} V_{0}(t(k))(1-d(k)) T_{s}\right.$

The discrete form of Eq. (26) can be expressed as

$i_{L}(k+1)=i_{L}(k)+\frac{V_{\text {in }}(k) T_{s}}{L}-\frac{V_{0}(k)(1-d(k)) T_{s}}{L}$

From Eq. (27), the inductor current of the next switching cycles is calculated by the inductor current from the present switching cycle, input voltage, output voltage and duty cycle. For calculating 


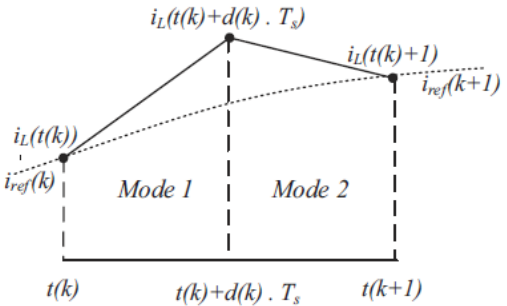

Fig. 2. Inductor current during one switching cycle.

the duty cycle $d(k)$, Eq. (27) can be rewritten as

$d(k)=\frac{L}{T_{s}} \frac{i_{L}(k+1)-i_{L}(k)}{V_{0}}+\frac{V_{0}(k)-V_{\text {in }}(k)}{V_{0}}$

Through the boost parameters such as input voltage, output voltage and inductor current, the duty cycle $d(k)$ for the actual switching cycle have been calculated. For the designed boost converter with PFC, the inductor current $i_{L}(k+1)$ has been forced to follow the reference $i_{\text {ref }}(k+1)$, which has a rectified sinusoidal form.

Substituting $V_{0}, i_{L}(k+1)$ in Eq. (28) by its references $V_{\text {ref }}$ and $i_{\text {ref }}(k+1)$ respectively, the duty cycle can be expressed as [12]:

$d(k)=\frac{L}{T_{s}} \cdot \frac{i_{\text {ref }}(k+1)-i_{L}(k)}{V_{\text {ref }}}+\frac{V_{\text {ref }}-V_{\text {in }}(k)}{V_{\text {ref }}}$

The reference current $i_{\text {ref }}$ in Eq. (29) is calculated as follow:

$i_{\text {ref }}(k+1)=I_{\text {max }} \cdot \mid \sin \left(\omega_{\text {line }} t(k+1) \mid\right.$

Where $I_{\max }$ is the peak value of the reference current, which is given by the output of the voltage loop controller.

All steps of the proposed method are shown in Fig. 3.

Step 1: Identification of the components parameters. Step 2: Determination of suitable voltage and introduce the HOSMC to regulate it.

Step 3: Determination of $I_{\text {Max }}$ through HOSMC, after that introduce the predictive control to regulate the current.

Step 4: Verify that all of duty cycles are calculated by the predictive control.

Step 5: Exploitation of the converter.

\section{Simulation results}

The aim of the simulation is to improve the performance of the power factor correction boost converter using HOSMC and predictive controllers to reduce the harmonic distortion produced by the nonlinear load and to achieve a unity power factor under parameters variation. The parameters of the system are presented in appendix. Due to the limitation of the paper presentation, only the performance of the proposed method is presented in simulation section. The performance of PI has been presented in experimental section.

Fig. 4 illustrates the source voltage, the output voltage, the source current simulated waveforms, for the proposed method at nominal load and nominal source voltage $(150 \mathrm{~V}, 100 \Omega)$ in the steady state. From this figure, it can be seen that the obtained results with the proposed control are satisfactory and require the international norms. The input current is in sinusoidal form and in phase with the source voltage. The total harmonic distortion (THD) is found less than $4 \%$, and the output voltage is maintained constant at desired value for a steady-state error of $1 \mathrm{~V}$.

In order to verify the robustness of the proposed method, the influence of the parameters changes (load resistor, reference

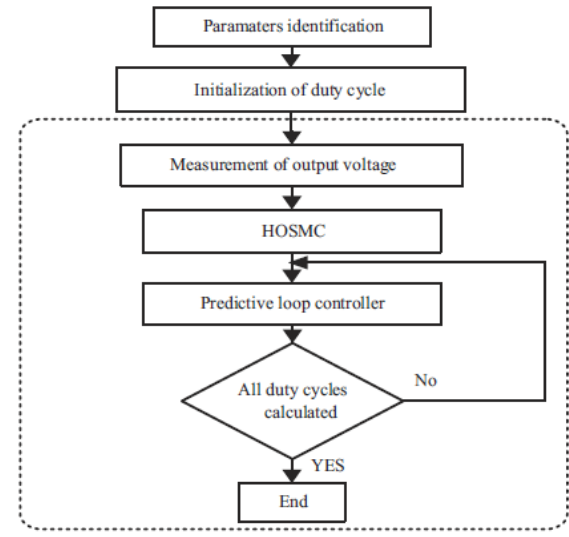

Fig. 3. Flowchart of proposed method.

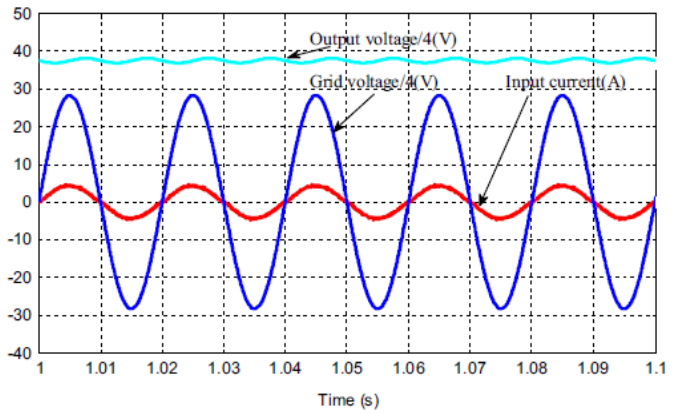

Fig. 4. Signal waveforms in the steady state.

output voltage) during steady and transient states based on the performance of the output voltage are studied by considering two cases.

1. Variation of $\pm 50 \%$ on load resistor.

2. Variation of $\pm 21 \%$ on reference output voltage.

Fig. 5 illustrates the transient response during the step change of $\mathrm{R}$ by keeping the reference output voltage fixed at $130 \mathrm{~V}$, the resistor load has been decreased $50 \%$ at $t=2 \mathrm{~s}$, and has been increased $50 \%$ at $t=4 \mathrm{~s}$, from $100 \Omega$ to $50 \Omega$ and from $50 \Omega$ to $100 \Omega$. After a short transient, the output voltage is maintained constant at its reference value during the load resistor variations, Also, from the figure an overshoot around $6 \mathrm{~V}(4.6 \%)$ with time response around $0.18 \mathrm{~s}$ in increasing load, and an overshoot around $7 \mathrm{~V}(5.38 \%)$ with response time around $0.16 \mathrm{~s}$ in decreasing load are observed. These results are much better compared to previous works (see Table 1).

Fig. 6 illustrates the step change of the reference output voltage, the reference output voltage has been increased $21 \%$ at $t=2 \mathrm{~s}$, and has been decreased $21 \%$ at $t=4 \mathrm{~s}$, from $140 \mathrm{~V}$ to $170 \mathrm{~V}$ and vice versa. After a short transient, the output voltage is maintained close to its reference with a short response time and a very little overshoot, from $140 \mathrm{~V}$ to $170 \mathrm{~V}$, where the response time is around $0.11 \mathrm{~s}$ with overshoot around $0.5 \mathrm{~V}$ (neglected). During the reference output voltage variation from $170 \mathrm{~V}$ to $140 \mathrm{~V}$ the response time is observed around $0.18 \mathrm{~s}$ with overshoot

\section{Link to full text articles :}

\section{http://www.ncbi.nlm.nih.gov/pubmed/25457043}


http://ac.els-cdn.com/S0019057814002535/1-s2.0-S0019057814002535- main.pdf? tid=886af4d0-4011-11e59595-00000aacb3628acdnat =1439288155_e141a40b22504a12118445665dd12506 